Special issue in honor of Prof. George C. Papageorgiou

\title{
TRIBUTE
}

\section{George C. Papageorgiou and the protective role of glycine betaine in activation and stabilization of the oxygen-evolving photosystem II complex}

\author{
N. MURATA* and K. STAMATAKIS ${ }^{* *}$ \\ National Institute for Basic Biology, 444-8585 Okazaki, Japan* \\ Institute of Biosciences and Applications, National Center for Scientific Research Demokritos, Aghia Paraskevi, \\ 15310 Attikis, Greece ${ }^{* *}$
}

\begin{abstract}
We present here our adventures in research in photosynthesis with George C. Papageorgiou (1933-2020) focusing on George's initiative in the discovery of the protective effects of glycine betaine on the oxygen-evolving photosystem II complex. We end with a brief description of research on glycine betaine-synthesizing transgenic cyanobacteria. Two of us, Norio Murata (in Japan) and Kostas Stamatakis (in Greece), and all our collaborators, have the highest respect for George, and we miss him and our intense discussions with him on various topics of photosynthesis research.
\end{abstract}

Keywords: glycine betaine; glycine betaine-synthesizing cyanobacteria; oxygen-evolving photosystem II complex.

\section{Discovery of the protective role of glycine betaine on the oxygen-evolving photosystem II complex}

Glycine betaine (N-trimethyl glycine) is a fully $\mathrm{N}$-methylsubstituted derivative of glycine, and is found in a large variety of microorganisms, higher plants, and animals; it belongs to a group of compounds, known collectively as 'compatible solutes' or 'cosolutes'; these small organic metabolites are very soluble in water and are nontoxic at high concentrations. Many halotolerant plants accumulate high amounts of glycine betaine in chloroplasts in response to abiotic stress, such as salt stress and cold stress (Weretilnyk et al. 1989, Sakamoto and Murata 2002). Glycine betaine effectively stabilizes the quaternary structures of enzymes and complex proteins, and it maintains the highly ordered state of membranes, at nonphysiological temperatures and salt concentrations (Papageorgiou and Murata 1995 and references therein).

George C. Papageorgiou, from the National Center for Scientific Research Demokritos, Greece, visited the National Institute of Basic Biology (NIBB) in Okazaki, Japan, for two months each in 1989 and 1990 (Fig. 1). During this period, he collaborated with one of us (Norio Murata). He was a theoretician with a strong background in biophysics and biochemistry. He hypothesized that quaternary ammonium cations would protect the PSII complex. He first tried a simple compound of quaternary ammonium cation, namely, choline, and performed a preliminary experiment, using PSII-containing thylakoid membranes. However, this compound was ineffective in

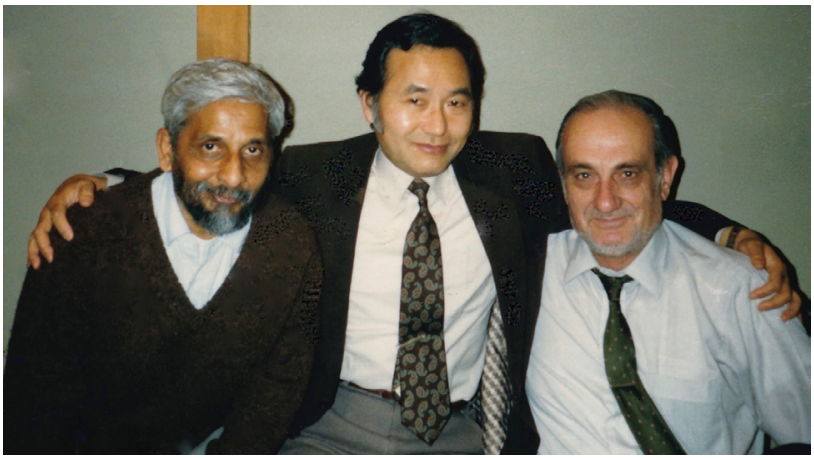

Fig. 1. Three talents of the glycine betaine research team. P.S. Mohanty (left), N. Murata (center), G.C. Papageorgiou (right). Taken at the National Institute for Basic Biology (NIBB), Okazaki, Japan, 1990

Received 13 September 2021

Accepted 15 November 2021

Published online 20 December 2021

${ }^{+}$Corresponding author

e-mail: kstam@bio.demokritos.gr

Conflict of interest: The authors declare that they have no conflict of interest. 
increasing the oxygen-evolving activity of PSII. Next, he tried glycine betaine, another compound of quaternary ammonium cation, and observed that this compound at 1.2 $\mathrm{M}$ protected the oxygen-evolving activity of PSII and prevented dissociation of extrinsic proteins of $23 \mathrm{kD}$ and $18 \mathrm{kD}$ (now termed PsbP and PsbQ, respectively) of the oxygen-evolving complex under salt stress conditions due to high concentrations of $\mathrm{NaCl}$.

George suggested that when the oxygen-evolving PSII complex was exposed to high concentrations of $\mathrm{NaC} 1$, the chaotropic action of $\mathrm{Cl}^{-}$anions would dissociate $\mathrm{PsbP}$ and PsbQ extrinsic proteins from the oxygen-evolving PSII complex, leading to the decline of the oxygen-evolving activity. In addition, he suggested that, in halotolerant plants living under saline conditions, $\mathrm{NaCl}$ would enter chloroplasts and cause the same effect as in the PSII complex in vitro. Further, glycine betaine, which was synthesized and accumulated within chloroplasts in these plants, would protect the oxygen-evolving PSII complex by preventing the dissociation of extrinsic proteins. This study was published in 1991, with the title 'Protection of the oxygen-evolving Photosystem II complex by glycinebetaine' (Papageorgiou et al. 1991).

\section{The second stage of research on the protective effect of glycine betaine}

After the above pioneering study by George, three researchers, Prasanna Mohanty (1934-2013, Jawaharlal Nehra University, India; see Papageorgiou G.C.: Prasanna K. Mohanty, 1 April, 1934 - 9 March, 2013. Photosynthetica 52: 481-483, 2014), Mahir D. Mamedov (Moscow State University, Russia), and Hidenori Hayashi (NIBB, Japan) joined the group to continue research on exploiting glycine betaine. The results of this research by George, with this team, are summarized below:

(1) Glycine betaine at $1.0 \mathrm{M}$ enhanced the oxygen-evolving activity of PSII by $40 \%$ and prevented its inactivation during incubation at room temperatures for a long time, up to even $6 \mathrm{~h}$. The enhancement and stabilization by glycine betaine were observed in the electron transport from $\mathrm{H}_{2} \mathrm{O}$ to dichlorophenol indophenol (DCPIP), but not from diphenyl carbazide (DPC) to DCPIP, suggesting that the action site of glycine betaine is specific to the oxygenevolving reaction, among all the reactions in PSII complex (Mamedov et al. 1991).

(2) Glycine betaine prevented not only the dissociation of the extrinsic proteins PsbP and PsbQ caused by the presence of $1.0 \mathrm{M} \mathrm{NaCl}$ but also the dissociation of the 33-kDa extrinsic protein (now termed PsbO) caused by 1.0 $\mathrm{M} \mathrm{MgCl}_{2}$ or $1.0 \mathrm{M} \mathrm{CaCl}_{2}$ (Murata et al. 1992).

(3) Glycine betaine protected the oxygen-evolving PSII complex, which had been depleted of all the three extrinsic proteins, against inactivation at high $\mathrm{pH}$ or during incubation at room temperature for a long time, up to even $4 \mathrm{~h}$. This result suggested that glycine betaine protected the oxygen-evolving complex per se in addition to the association of extrinsic proteins to it (Mohanty et al. 1993). (4) Glycine betaine protected the oxygen-evolving PSII complex in thylakoid membranes, which had been prepared from the cyanobacterium Phormidium laminosum, against both salt and heat stress (Stamatakis and Papageorgiou 1993).

In summarizing this series of research, George and one of us (Norio Murata) published in Photosynthesis Research a review entitled 'The unusually strong stabilizing effects of glycinebetaine on the structure and function of the oxygen-evolving Photosystem II complex' (Papageorgiou and Murata 1995). This review has been highly recognized, as evidenced by its high citation, for example, 500 in Google Scholar. In this review, George strongly advised that researchers should add 1.0 1.5 M glycine betaine in all media of preparation, storage, and assay of oxygenevolving PSII preparations to achieve the maximum activity of oxygen evolution and its stabilization. Many researchers have followed his advice and have obtained great success (Eshaghi et al. 1999, Schiller and Dau 2000, Allakhverdiev et al. 2003). Generation of glycine betaine-synthesizing transgenic
cyanobacteria and plants at NIBB, Japan

Impact of the protective effect of glycine betaine on the oxygen-evolving PSII complex opened a new series of studies of glycine betaine, i.e., generation of transgenic cyanobacteria and plants that synthesize glycine betaine in vivo. A survey of the literature led us to use choline oxidase to be a target enzyme for the synthesis of glycine betaine; this enzyme is present in a soil bacterium Arthrobacter globiformis and is active in converting choline to glycine betaine by an oxygenation reaction. Researchers in Norio Murata's laboratory isolated the $\operatorname{cod} A$ gene for this enzyme and transformed the cyanobacterium Anacystis nidulans (Synechococcus elongatus sp. PCC 7942) with this gene. The resultant transgenic cells synthesized glycine betaine at $60-80 \mathrm{mM}$ within cells and revealed extensive improvement of tolerance to salt and cold stresses (Deshnium et al. 1995, 1997). This research, with the same gene, was then extended to a higher plant Arabidopsis thaliana, and the NIBB team observed that the resultant transgenic plants were much more tolerant than the control plants under abiotic stress, such as high-salt, cold, heat, and high-light stress. These studies have been summarized in several reviews (for example, Sakamoto and Murata 2001, Chen and Murata 2002).

Continuous research over the years has transformed various crop plants, along with model plants, by introducing bacterial or plant genes for the synthesis of glycine betaine. The resultant glycine betaine-synthesizing crop plants exhibit enhanced tolerance to various abiotic stresses and out-yield wild-type plants under stressful conditions (Chen and Murata 2011, Ahmad et al. 2013).

\section{Research on the glycine betaine-synthesizing transgenic cyanobacterium at the National Center for Scientific Research Demokritos, Greece}

One of us (Kostas Stamatakis) joined the laboratory of George C. Papageorgiou in 1995 and, in 2000, became the head of the laboratory (Fig. 2). Before joining George, Kostas had studied the protective role of glycine betaine 


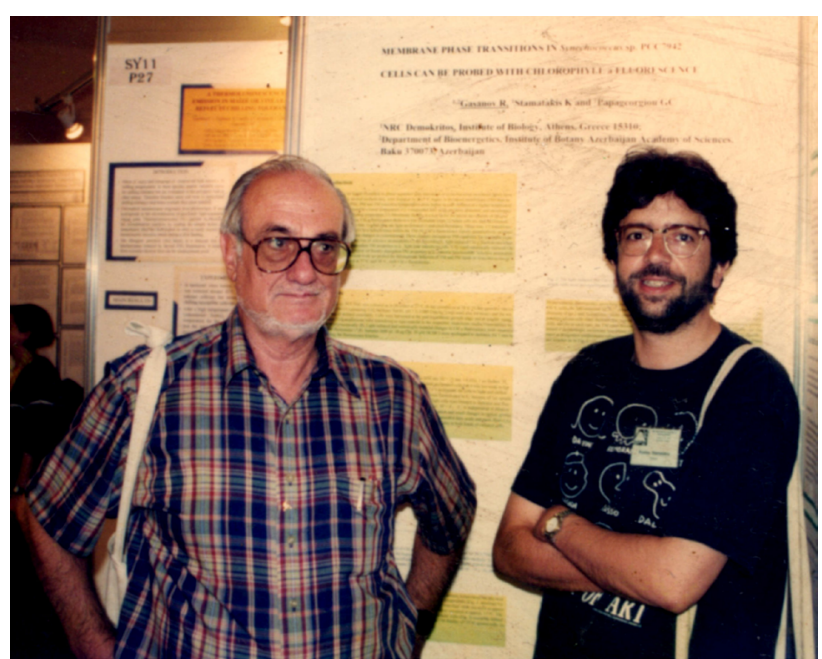

Fig. 2. Two excellent leaders of the photosynthesis research in National Center for Scientific Research Demokritos. George C. Papageorgiou (left) and Kostas Stamatakis (right). Taken at the $11^{\text {th }}$ International Congress on Photosynthesis, Budapest, 1998.

in the activation of phosphoenolpyruvate carboxylase (Stamatakis et al. 1988). George and Kostas studied the $\operatorname{cod} A$-transgenic cyanobacterium at the National Center for Scientific Research Demokritos whereas members in Murata's laboratory (NIBB, Japan) studied the codA-transgenic Arabidopsis thaliana. George, and his Ph.D. student Nectarios P. Ladas, together with Kostas, used the $\operatorname{cod} A$-transgenic glycine betaine-synthesizing Synechococcus elongatus and studied cell turgor and cell proliferation under salt-stress conditions. They showed that glycine betaine, present in transgenic cells, improved their tolerance to salinity by activating sucrose biosynthesis and by alleviating sucrose toxicity, leading to their high rate of proliferation (Ladas et al. 1998, Ladas and Papageorgiou 2000). However, it is still uncertain whether the increase in the internal osmolality from 0.65 to $1.0 \mathrm{Osm} \mathrm{kg} \mathrm{kg}^{-1}$ sustains turgor pressure to allow growth in the presence of salt. Recently, the research group of Kostas, together with George, discovered that the $\operatorname{cod} A$-transgenic cyanobacterium could be used as a 'cell factory' to produce sucrose and $\mathrm{H}_{2}$ under salt-stress and anaerobic conditions (Vayenos et al. 2020). However, it is notable that the effective concentration of glycine betaine for protection against salt stress is $1.0-1.5 \mathrm{M}$ in isolated PSII, whereas it is $60-80 \mathrm{mM}$ in cyanobacterial cells. It appears that the mechanism of protection by glycine betaine is somewhat different between the in vitro and in vivo conditions.

\section{George Papageorgiou was a loving person with a pleasant and joyful personality}

Norio Murata had enjoyed a pleasant time with George during his stay in the NIBB in Okazaki as well as on many occasions at other places around the world. In Okazaki, George liked to visit a style of Japanese grill 'robatayaki', where uncooked food items are placed on display in front of the customers, who could then order any of the items as well as the way of cooking. George remarked that 'robatayaki' was similar to the Greek-style restaurant 'taverna'. He visited this restaurant not only with other members of the laboratory, but many times alone, and made friends there. His joyful personality was appreciated by members and visitors of the laboratory. Sadly, they are unable to see and talk to George with a glass of beer and sake anymore! We all miss him.

\section{References}

Ahmad R., Lim C.J., Kwon S.-Y.: Glycine betaine: a versatile compound with great potential for gene pyramiding to improve crop plant performance against environmental stresses. - Plant Biotechnol. Rep. 7: 49-57, 2013.

Allakhverdiev S.I., Hayashi H., Nishiyama Y. et al: Glycinebetaine protects the D1/D2/Cytb559 complex of photosystem II against photo-induced and heat-induced inactivation. - J. Plant Physiol. 160: 41-49, 2003.

Chen T.H.H., Murata N.: Enhancement of tolerance to abiotic stress by metabolic engineering of betaines and other compatible solutes. - Curr. Opin. Plant Biol. 5: 250-257, 2002.

Chen T.H.H., Murata N.: Glycinebetaine protects plants against abiotic stress: Mechanisms and biotechnological applications. - Plant Cell Environ. 34: 1-20, 2011.

Deshnium P., Gombos Z., Nishiyama Y., Murata N.: The action in vivo of glycine betaine in enhancement of tolerance of Synechococcus sp. strain PCC 7942 to low temperatures. J. Bacteriol. 179: 339-344, 1997.

Deshnium P., Los D.A., Hayashi H. et al.: Transformation of Synechococcus with a gene for choline oxidase enhances tolerance to salt stress. - Plant Mol. Biol. 29: 897-907, 1995.

Eshaghi S., Andersson B., Barber J.: Isolation of a highly active PSII-LHCII supercomplex from thylakoid membranes by a direct method. - FEBS Lett. 446: 23-26, 1999.

Ladas N.P., Papageorgiou G.C.: Cell turgor: a critical factor for the proliferation of cyanobacteria at unfavorable salinity. Photosynth. Res. 65: 155-164, 2000.

Ladas N.P., Stamatakis K., Papageorgiou G.C.: Critical role of turgor pressure for the proliferation of Synechococcus sp. PCC7942 at unfavorable salinity. - In: Garab G. (ed.): Photosynthesis: Mechanisms and Effects. Pp. 2605-2608. Springer, Dordrecht 1998.

Mamedov M.D., Hayashi H., Wada H. et al.: Glycinebetaine enhances and stabilizes the evolution of oxygen and the synthesis of ATP by cyanobacterial thylakoid membranes. FEBS Lett. 294: 271-274, 1991.

Mohanty P., Hayashi H., Papageorgiou G.C., Murata N.: Stabilization of the Mn-cluster of the oxygen-evolving complex by glycinebetaine. - BBA-Bioenergetics 1144: 9296, 1993.

Murata N., Mohanty P.S., Hayashi H., Papageorgiou G.C.: Glycinebetaine stabilizes the association of extrinsic proteins with the photosynthetic oxygen-evolving complex. - FEBS Lett. 296: 187-189, 1992.

Papageorgiou G.C., Fujimura Y., Murata N.: Protection of the oxygen-evolving photosystem II complex by glycinebetaine.BBA-Bioenergetics 1057: 361-366, 1991.

Papageorgiou G.C., Murata N.: The unusually strong stabilizing effect of glycinebetaine on the structure and function of the oxygen-evolving photosystem II complex. - Photosynth. Res. 44: 243-252, 1995.

Sakamoto A., Murata N.: The use of bacterial choline oxidase, a glycinebetaine-synthesizing enzyme, to create stress-resistant 
transgenic plants. - Plant Physiol. 125: 180-188, 2001.

Sakamoto A., Murata N.: The role of glycine betaine in the protection of plants from stress: Clues from transgenic plants. - Plant Cell Environ. 25: 163-171, 2002.

Schiller H., Dau H.: Preparation protocols for high-activity Photosystem II membrane particles of green algae and higher plants, $\mathrm{pH}$ dependence of oxygen evolution and comparison of the $\mathrm{S}_{2}$-state multiline signal by X-band EPR spectroscopy. J. Photoch. Photobio. B 55: 138-144, 2000.

Stamatakis C., Papageorgiou G.C.: Stabilization of photosystem II particles isolated from the thermophilic cyanobacterium Phormidium laminosum with glycinebetaine and glycerol. BBA-Bioenergetics 1183: 333-338, 1993.
Stamatakis K., Gavalas N.A., Manetas Y.: Organic cosolutes increase the catalytic efficiency of phosphoenolpyruvate carboxylase, from Cynodon dactylon (L.) Pers., apparently through self-association of the enzymic protein. - Aust. J. Plant Physiol. 15: 621-631, 1988.

Vayenos D., Romanos G.E., Papageorgiou G.C., Stamatakis K. Synechococcus elongatus PCC7942: a cyanobacterium cell factory for producing useful chemicals and fuels under abiotic stress conditions. - Photosynth. Res. 146: 235-245, 2020.

Weretilnyk E.A., Bednarek S., McCue K.F. et al.: Comparative biochemical and immunological studies of the glycine betaine synthesis pathway in diverse families of dicotyledons. Planta 178: 342-352, 1989.

(C) The authors. This is an open access article distributed under the terms of the Creative Commons BY-NC-ND Licence. 